\title{
CONDITIONS ON THE LOGARITHMIC DERIVATIVE OF A FUNCTION IMPLYING BOUNDEDNESS
}

\author{
T. H. MACGREGOR AND F. RØNNING
}

\begin{abstract}
In this paper we investigate functions analytic and nonvanishing in the unit disk, with the property that the logarithmic derivative is contained in some domain $\Omega$. We obtain conditions on $\Omega$ which imply that the functions are bounded and that their first derivatives belong to $H^{p}$ for some $p \geq 1$. For certain domains $\Omega$ the sufficient conditions that we give are also, in some sense, necessary. Examples of domains to which the results apply are given.
\end{abstract}

\section{INTRODUCTION}

Let $\Delta=\{z:|z|<1\}$, and let $\mathscr{A}$ be the set of functions that are analytic in $\Delta$. The main purpose of this paper is to formulate conditions on the logarithmic derivative of a nonvanishing function in $\mathscr{A}$ which imply that the modulus of the function is bounded from above and below. We also obtain conditions from which it follows that the function's derivative belongs to the Hardy space $H^{p}$. The results tie in with the theory of starlike univalent functions which are characterized in terms of the logarithmic derivative. In that context we usually adopt the normalization $f(0)=f^{\prime}(0)-1=0$, and then the class $S^{*}$ of functions starlike with respect to the origin is characterized by

$$
f \in S^{*} \Longleftrightarrow \operatorname{Re} \frac{z f^{\prime}(z)}{f(z)} \geq 0, \quad z \in \Delta .
$$

The functions in $S^{*}$ are in general not bounded, and a typical example of an unbounded function in $S^{*}$ is given by the Koebe function $k(z)=z /(1-z)^{2}$. Well-known subclasses of $S^{*}$ are the functions starlike of order $\alpha, S_{\alpha}^{*}$, given by

$$
f \in S_{\alpha}^{*} \Longleftrightarrow \operatorname{Re} \frac{z f^{\prime}(z)}{f(z)} \geq \alpha, \quad 0 \leq \alpha \leq 1, \quad z \in \Delta,
$$

and the strongly starlike functions, $S S_{\alpha}^{*}$, given by

$$
f \in S S_{\alpha}^{*} \Longleftrightarrow\left|\arg \frac{z f^{\prime}(z)}{f(z)}\right| \leq \frac{\pi \alpha}{2}, \quad 0 \leq \alpha \leq 1, \quad z \in \Delta .
$$

For $\alpha<1$ the class $S_{\alpha}^{*}$ contains unbounded functions, and a typical example is $k_{\alpha}(z)=z /(1-z)^{2-2 \alpha}$; whereas all functions in $S S_{\alpha}^{*}, \alpha<1$, are known

Received by the editors April 12, 1994; originally communicated to the Proceedings of the AMS by Albert Baernstein.

1991 Mathematics Subject Classification. Primary 30C35; Secondary 30C20, 30C45, 30D55.

The research of the second author was partially supported by a grant from the Nansen Foundation. 
to be bounded [1]. The same is true if the wedge in (1.1) is replaced by a parabola with vertex at the origin and axis along the positive real axis [6]. In [5] the question was raised whether all bounded starlike functions have an integral representation

$$
f(z)=\int_{|x|=1} \log \frac{1}{1-x z} d \mu(x)+f(0)
$$

for some complex-valued measure $\mu$ on the unit circle. The investigations in this paper to some extent also relate to this question.

\section{THE MAIN RESUlTS}

Before we get to the results we shall describe the basic setup for the whole work. Suppose that $f$ is a function which is analytic and nonzero in $\Delta$, and let $g(z)=f^{\prime}(z) / f(z)$. Assume that $g(z) \in \Omega$ where $\Omega$ is a simply connected domain and $\Omega \neq \mathbb{C}$. Let $w_{0}=g(0)$, and let $G$ be a conformal mapping of $\Delta$ onto $\Omega$ such that $G(0)=w_{0}$. Under these assumptions we can prove the following results which will be used later.

Lemma 2.1. Let $M(r)=\max _{|z| \leq r}|G(z)|$ for $0<r<1$.

(a) If

$$
\int_{0}^{1} M(t) d t<\infty
$$

then $f$ and $1 / f$ are bounded functions.

(b) If

$$
\int_{0}^{1} M^{p}(t) d t<\infty
$$

for some $p \geq 1$, then $f^{\prime} \in H^{p}$.

Proof. Clearly $g \prec G$, so we have

$$
\max _{|z| \leq r}|g(z)| \leq \max _{|z| \leq r}|G(z)|
$$

for $0<r<1$. Because $M$ is nondecreasing, this implies that

$$
\int_{0}^{1}|g(t z)| d t \leq \int_{0}^{1} M(t|z|) d t \leq \int_{0}^{1} M(t) d t .
$$

Therefore (2.1) implies that

$$
\sup _{|z|<1} \int_{0}^{1}|g(t z)| d t<\infty .
$$

We have $\log (f(z) / f(0))=\int_{C} g(w) d w$ where $C$ is the line segment from 0 to $z$. Hence

$$
\left|\log \frac{f(z)}{f(0)}\right|=\left|z \int_{0}^{1} g(t z) d t\right| \leq \int_{0}^{1}|g(t z)| d t .
$$

Thus equation (2.3) implies that $\sup _{|z|<1}|\log (f(z) / f(0))|$ is finite and hence $\sup _{|z|<1}|\log (|f(z) / f(0)|)|$ is finite, and the conclusion of part (a) follows.

To prove part (b) note that (2.2) implies (2.1) since $p \geq 1$ and therefore $f$ is bounded. Because $g(z)=f^{\prime}(z) / f(z)$, we have $\left|f^{\prime}(z)\right| \leq\|f\|_{H^{\infty}}|g(z)|$, and 
hence, $f^{\prime} \in H^{p}$ if we can show that $g \in H^{p}$. By Littlewood's subordination principle we get

$$
\frac{1}{2 \pi} \int_{0}^{2 \pi}\left|g\left(r e^{i \theta}\right)\right|^{p} d \theta \leq \frac{1}{2 \pi} \int_{0}^{2 \pi}\left|G\left(r e^{i \theta}\right)\right|^{p} d \theta
$$

for $0<r<1$, so it suffices to prove that $G \in H^{p}$. Let $K(z)=G(z)-G(0)$ and $N(r)=\max _{|z| \leq r}|K(z)|$. Then $N(r) \leq M(r)+|G(0)|$. Since $K$ is analytic and univalent in $\Delta$ and $K(0)=0$, we can use Prawitz's theorem [3, p. 61] to get

$$
\frac{1}{2 \pi} \int_{0}^{2 \pi}\left|K\left(r e^{i \theta}\right)\right|^{p} d \theta \leq p \int_{0}^{1} \frac{1}{t} N^{p}(t) d t .
$$

For $a \geq 0$ and $b \geq 0$ there is a constant $A_{p}$, depending only on $p$, such that $(a+b)^{p} \leq A_{p}\left(a^{p}+b^{p}\right)$. Therefore,

$$
\begin{aligned}
\frac{1}{2 \pi} \int_{0}^{2 \pi}\left|G\left(r e^{i \theta}\right)\right|^{p} d \theta & \leq A_{p}\left[\frac{1}{2 \pi} \int_{0}^{2 \pi}\left|K\left(r e^{i \theta}\right)\right|^{p} d \theta+|G(0)|^{p}\right] \\
& \leq A_{p}\left[p \int_{0}^{1} \frac{1}{t} N^{p}(t) d t+|G(0)|^{p}\right] .
\end{aligned}
$$

Because $N(r) \leq M(r)+|G(0)|$ and $K(0)=0$, Schwarz's lemma implies that $N(r) \leq r(M(r)+|G(0)|)$. Thus

$$
\begin{aligned}
\frac{1}{2 \pi} \int_{0}^{2 \pi}\left|G\left(r e^{i \theta}\right)\right|^{p} d \theta & \leq A_{p}\left[p \int_{0}^{1} t^{p-1}(M(t)+|G(0)|)^{p} d t+|G(0)|^{p}\right] \\
& \leq A_{p}\left[p \int_{0}^{1}(M(t)+|G(0)|)^{p} d t+|G(0)|^{p}\right] \\
& \leq A_{p}\left[p A_{p}\left(\int_{0}^{1} M^{p}(t) d t+|G(0)|^{p}\right)+|G(0)|^{p}\right] .
\end{aligned}
$$

This shows that (2.2) implies $G \in H^{p}$.

In the following we further assume that $\Omega$ is an unbounded Jordan domain and that the origin is on the boundary of $\Omega$. Then some point $z_{\infty}$ on $\partial \Delta$ corresponds to $\infty$ under the mapping $G$, and some other point, $z_{0}$, corresponds to 0 . By a rotation in $z$ we can assume that $z_{\infty}=1$. The point 0 breaks up $\partial \Omega$ into two Jordan curves, which we denote $\Gamma^{+}$and $\Gamma^{-}$, each having endpoints 0 and $\infty$. Let $\Gamma$ be a Jordan curve with endpoints 0 and $\infty$ all of whose other points belong to $\Omega$. For each $R>0$ there is an arc denoted $\gamma_{R}$ which is on $\Omega \cap\{w:|w|=R\}$, meets $\Gamma$, and has one endpoint on $\Gamma^{+}$and the other on $\Gamma^{-}$. Let $\ell(R)$ denote the length of $\gamma_{R}$, and let $\varphi(R)=\ell(R) / R$. Then $\varphi(R)$ gives the angular variation of $\gamma_{R}$. We have $0 \leq \varphi(R) \leq 2 \pi$ and $0 \leq \ell(R) \leq 2 \pi R$. Now we are ready to state the main theorem about boundedness of the function $f$.

Theorem 2.2. Let $\Omega$ and $\varphi$ be as described above. Let $f \in \mathscr{A}, f(z) \neq 0$ for $z \in \Delta$, and assume $g(\Delta) \subset \Omega$ where $g(z)=f^{\prime}(z) / f(z)$. If

$$
\int_{1}^{\infty} \frac{d u}{\varphi(u) \exp \left\{\pi \int_{1}^{u} \frac{d x}{x \varphi(x)}\right\}}<\infty,
$$


then there are positive constants $m$ and $M$ such that

$$
m \leq|f(z)| \leq M, \quad z \in \Delta .
$$

Proof. The proof basically involves setting up a suitable composition of conformal mappings, so the Ahlfors' distortion theorem (see [4, p.136]) can be used. Recall that $G(\Delta)=\Omega, G\left(z_{0}\right)=0$, and $G(1)=\infty$. There are complex numbers $x$ and $\alpha$ such that $|x|=1,|\alpha|<1$, and the function

$$
\omega(z)=x \frac{z+\alpha}{1+\bar{\alpha} z}, \quad|z|<1,
$$

satisfies $\omega(1)=1$ and $\omega\left(z_{0}\right)=-1$. An easy computation shows that there are positive constants $A$ and $B$, depending only on $\alpha$, such that

$$
A \leq \frac{1-|\omega(z)|}{1-|z|} \leq B
$$

for $|z|<1$. Indeed we can let $A=(1-|\alpha|) / 4$ and $B=4 /(1-|\alpha|)$. Write $w=G(z), \zeta=\log w$, and $\sigma=\log ((1+\omega(z)) /(1-\omega(z)))$. Then $z \mapsto \sigma$ is a conformal mapping of $\Delta$ onto the strip $\mathscr{S}=\{\sigma:|\operatorname{Im} \sigma|<\pi / 2\}$. Also $w \mapsto \zeta$ is a conformal mapping of $\Omega$ onto a domain $\Phi$. The domain $\Phi$ is unbounded, and each line $\{\zeta: \operatorname{Re} \zeta=$ constant $\}$ meets $\Phi$. The arc $\gamma_{R}$ corresponds to a line segment denoted $\theta_{s}$ where $s=\operatorname{Re} \zeta=\log R$. If we let $\theta(s)$ denote the length of $\theta_{s}$, then $\theta(s)=\varphi(R)$. The boundary of $\Phi$ consists of two Jordan $\operatorname{arcs} \Lambda^{+}$and $\Lambda^{-}$having endpoints at $\infty$. Through the composition of the mappings described above we obtain a mapping $\sigma=\sigma(\zeta)$ of $\Phi$ onto $\mathscr{S}$ such that $-\infty$ corresponds to $-\infty$ and $\infty$ corresponds to $\infty$. We can apply the Ahlfors' distortion theorem to this mapping. Hence if $a_{0}<a$ and

$$
\int_{a_{0}}^{a} \frac{1}{\theta(s)} d s>2
$$

then

$$
\operatorname{Re}\left[\sigma(\zeta)-\sigma\left(\zeta_{0}\right)\right]>\pi \int_{a_{0}}^{a} \frac{1}{\theta(s)} d s-4 \pi,
$$

for $\zeta_{0} \in \theta_{a_{0}}$ and $\zeta \in \theta_{a}$. We fix $a_{0}$ and choose a point $\zeta_{0} \in \theta_{a_{0}}$. Then for $z$ sufficiently near 1 the corresponding $\zeta$ satisfies $\zeta \in \theta_{a}$ and $a>a_{0}$. Let $R_{0}=e^{a_{0}}$. Then

$$
\int_{a_{0}}^{a} \frac{1}{\theta(s)} d s=\int_{R_{0}}^{R} \frac{1}{x \varphi(x)} d x \geq \int_{R_{0}}^{R} \frac{1}{2 \pi x} d x=\frac{1}{2 \pi} \log \frac{R}{R_{0}} .
$$

Hence (2.6) is satisfied for all $z$ sufficiently near 1 . Since

$$
\sigma(\zeta)=\log ((1+\omega(z)) /(1-\omega(z)))
$$

(2.5) implies

$$
\operatorname{Re} \sigma(\zeta)=\log \left|\frac{1+\omega(z)}{1-\omega(z)}\right| \leq \log 2+\log \frac{1}{1-|\omega(z)|} \leq \log 2-\log A+\log \frac{1}{1-r}
$$

where $r=|z|$. Hence (2.7) implies

$$
\int_{a_{0}}^{a} \frac{1}{\theta(s)} d s \leq \frac{1}{\pi} \log \frac{1}{1-r}+C
$$


for some constant $C$. This is the same as

$$
\int_{R_{0}}^{R} \frac{1}{x \varphi(x)} d x \leq \frac{1}{\pi} \log \frac{1}{1-r}+C .
$$

Hence (2.8) holds for all $z$ sufficiently near 1 , where $r=|z|$ and $R=|G(z)|$. For $0 \leq r<1$, let $u=u(r)$ be defined by the equality

$$
\int_{R_{0}}^{u} \frac{1}{x \varphi(x)} d x=\frac{1}{\pi} \log \frac{1}{1-r}+C .
$$

Since $1 / x \varphi(x)>0$, this uniquely determines $u$. Moreover $u(r)>R_{0}$ and (2.8) and (2.9) imply that $R=|G(z)| \leq u(r)$ for $|z|=r$ and for all $z$ sufficiently near 1 . For every $\varepsilon(0<\varepsilon<2) G$ is bounded in $\Delta \backslash\{z:|z-1| \geq \varepsilon\}$. Hence if we have

$$
\int_{0}^{1} u(r) d r<\infty
$$

then $\int_{0}^{1} M(r) d r<\infty$ and by Lemma 2.1 (a) we have that $f$ and $1 / f$ are bounded.

What remains to establish the theorem is therefore to prove that the condition (2.4) implies (2.10). Let $R_{1}$ be defined by $\int_{R_{0}}^{R_{1}} \frac{1}{x \varphi(x)} d x=C$, where $C$ is as in (2.9). Then

$$
\int_{0}^{1} u(r) d r=\int_{R_{1}}^{\infty} u \frac{d r}{d u} d u
$$

From (2.9) we get

$$
\frac{d r}{d u}=\frac{\pi e^{C \pi}}{u \varphi(u) \exp \left\{\pi \int_{R_{0}}^{u} \frac{d x}{x \varphi(x)}\right\}}
$$

and further,

$$
\int_{0}^{1} u(r) d r=\pi e^{C \pi} \int_{R_{1}}^{\infty} \frac{d u}{\varphi(u) \exp \left\{\pi \int_{R_{0}}^{u} \frac{d x}{x \varphi(x)}\right\}} .
$$

We now see that (2.4) implies (2.10).

The examples of starlike functions mentioned in the introduction might suggest that boundedness or nonboundedness is connected to how close $\Omega$ is to a halfplane. Indeed it is the case that the integral in (2.4) may exist or not exist depending on how fast the function $\varphi(u)$ tends to $\pi$ when $u \rightarrow \infty$. We will give examples illustrating this in the last section. In order to obtain, in some sense, a maximal domain $\Omega$ which yields $f$ and $1 / f$ bounded, it is therefore natural to put further assumptions on $\varphi$, e.g., that it is monotonically increasing to $\pi$. Under such assumptions the condition (2.4) can be replaced by simpler conditions.

Theorem 2.3. Let $\Omega, \varphi, f$, and $g$ be as in Theorem 2.2.

(a) Assume that $\lim _{u \rightarrow \infty} \varphi(u)=\pi$. If

$$
\int_{1}^{\infty} \frac{d u}{\exp \left\{\pi \int_{1}^{u} \frac{d x}{x \varphi(x)}\right\}}<\infty,
$$

then $f$ and $1 / f$ are bounded. 
(b) Assume that $\lim _{u \rightarrow \infty} \varphi(u)=\pi$ and that for some $\alpha \geq 1$ the function $\varphi$ is nondecreasing in $[\alpha, \infty\rangle$. If

$$
\int_{1}^{\infty} \frac{d u}{u^{\pi / \varphi(u)}}<\infty
$$

then $f$ and $1 / f$ are bounded.

Proof. Since $\lim _{u \rightarrow \infty} \varphi(u)=\pi$ then $1 / \varphi(u)$ is bounded for $u$ large enough, and part (a) follows from Theorem 2.2. Assume also that $\varphi$ is nondecreasing in $[\alpha, \infty\rangle$ for some $\alpha \geq 1$. Then

$$
\int_{\alpha}^{u} \frac{1}{x \varphi(x)} d x \geq \frac{\log u}{\varphi(u)}-\frac{\log \alpha}{\varphi(\alpha)} .
$$

Hence $\exp \left\{-\pi \int_{\alpha}^{u} \frac{1}{x \varphi(x)} d x\right\} \leq D u^{-\pi / \varphi(u)}$ for some constant $D$. Therefore (2.13) implies (2.12) and part (b) follows.

We now turn to the discussion of conditions which imply that the derivative of the function $f$ belongs to some Hardy space $H^{p}$. The arguments used here will be parallel to those used for proving boundedness. Assume that $\Omega$ has the properties described before Theorem 2.2, and let $f, g, G$, and $\varphi$ be as before. Also let $u$ be defined as in (2.9). Then it is easy to see that

$$
\int_{0}^{1} u^{p}(r) d r<\infty, \quad p \geq 1,
$$

implies (2.2) just as (2.10) implies (2.1). Thus the conditions we pose will be such that (2.14) holds, and therefore by Lemma 2.1(b) we conclude that $f^{\prime} \in H^{p}$.

Theorem 2.4. Let $\Omega$ and $\varphi$ be as described before Theorem 2.2. Let $f \in \mathscr{A}$ and $f(z) \neq 0$ for $z \in \Delta$, and assume $g(\Delta) \subset \Omega$ where $g(z)=f^{\prime}(z) / f(z)$. If

$$
\int_{1}^{\infty} \frac{u^{p-1} d u}{\varphi(u) \exp \left\{\pi \int_{1}^{u} \frac{d x}{x \varphi(x)}\right\}}<\infty
$$

for some $p \geq 1$, then $f^{\prime} \in H^{p}$.

Proof. The argument leading to (2.11) yields

$$
\int_{0}^{1} u^{p}(r) d r=\pi e^{C \pi} \int_{R_{1}}^{\infty} \frac{u^{p-1} d u}{\varphi(u) \exp \left\{\pi \int_{R_{0}}^{u} \frac{d x}{x \varphi(x)}\right\}},
$$

so (2.15) implies (2.14) and the proof is complete.

Under additional assumptions on $\varphi$ it is clear that we can obtain a theorem parallel to Theorem 2.3.

Theorem 2.5. Let $\Omega, \varphi, f$, and $g$ be as in Theorem 2.4.

(a) Assume that $\lim _{u \rightarrow \infty} \varphi(u)=\pi / p, p \geq 1$. If

$$
\int_{1}^{\infty} \frac{u^{p-1} d u}{\exp \left\{\pi \int_{1}^{u} \frac{d x}{x \varphi(x)}\right\}}<\infty
$$

then $f^{\prime} \in H^{p}$. 
(b) Assume that $\lim _{u \rightarrow \infty} \varphi(u)=\pi / p, p \geq 1$, and that for some $\alpha \geq 1 \varphi$ is nondecreasing in $[\alpha, \infty)$. If

$$
\int_{1}^{\infty} \frac{d u}{u^{1-p+\pi / \varphi(u)}}<\infty
$$

then $f^{\prime} \in H^{p}$.

\section{CONVERSE VERSIONS OF THE RESULTS IN SECTION 2}

The basic setup remains the same as it has been. Under certain additional assumptions on the domain $\Omega$, expressed in terms of $\varphi$, we can prove results converse to Theorem 2.2 and Theorem 2.4. The basic tool here is a theorem of Warschawski [4, p. 140] which we state below. With the same notation as in the proof of Theorem 2.2, suppose that the curves $\Lambda^{+}$and $\Lambda^{-}$have the form $y=\tau^{+}(x)$ and $y=\tau^{-}(x)$, where $\tau^{+}>\tau^{-}$. Then $\theta(x)=\tau^{+}(x)-\tau^{-}(x)$, and we define $\tau(x)=\frac{1}{2}\left(\tau^{+}(x)+\tau^{-}(x)\right)$.

Warschawski's theorem. Suppose that for all $x$ we have $\left|\tau^{\prime}(x)\right|<M,\left|\theta^{\prime}(x)\right|<$ $M$. If $a_{0}<a, \zeta_{0} \in \theta_{a_{0}}, \zeta \in \theta_{a}$, then

$$
\operatorname{Re}\left[\sigma(\zeta)-\sigma\left(\zeta_{0}\right)\right] \leq \pi \int_{a_{0}}^{a} \frac{1+\tau^{\prime 2}(x)}{\theta(x)} d x+\frac{\pi}{12} \int_{a_{0}}^{a} \frac{\theta^{\prime 2}(x)}{\theta(x)} d x+B,
$$

where $B$ is a constant depending on $M$.

Let $\Omega$ be as before, and in addition assume that it is symmetric about the real axis. Also assume that there is a constant $M$ such that $\left|r \varphi^{\prime}(r)\right| \leq M$. With these assumptions we have $\tau(x) \equiv 0$ and $\left|\theta^{\prime}(x)\right| \leq M$, so the Warschawski theorem can be applied to get

$$
\operatorname{Re}\left[\sigma(\zeta)-\sigma\left(\zeta_{0}\right)\right] \leq \pi \int_{a_{0}}^{a} \frac{1}{\theta(x)} d x+\frac{\pi}{12} \int_{a_{0}}^{a} \frac{\theta^{\prime 2}(x)}{\theta(x)} d x+B .
$$

Now $\int_{a_{0}}^{a} \frac{\theta^{\prime 2}(x)}{\theta(x)} d x \leq M\left(\log \theta(a)-\log \theta\left(a_{0}\right)\right)$ which is bounded by an absolute constant, so for some $C>0$ we have

$$
\operatorname{Re}\left[\sigma(\zeta)-\sigma\left(\zeta_{0}\right)\right] \leq \pi \int_{a_{0}}^{a} \frac{1}{\theta(x)} d x+C .
$$

Choose $G$, analytic and univalent in $\Delta$, such that $G(\Delta)=\Omega, G(1)=\infty$, and $G(-1)=0$. For real $r$ close to 1 we now have, in the same way as (2.8) was proved,

$$
\int_{R_{0}}^{R} \frac{1}{x \varphi(x)} d x \geq \frac{1}{\pi} \log \frac{1}{1-r}-C .
$$

With $u=u(r)$ defined such that equality holds in (3.1) we get

$$
u(r) \leq R=|G(z)| \leq M(r)
$$

and so $\int_{0}^{1} u(r) d r=\infty$ implies $\int_{0}^{1} M(r) d r=\infty$. Define $F$ by $F^{\prime}(z) / F(z)=$ $G(z)$. From the assumptions on $\Omega$ it follows that $M(r)=G(r)$, so we have

$$
\lim _{r \rightarrow 1}\left|\log \frac{F(r)}{F(0)}\right|=\lim _{r \rightarrow 1}\left|\int_{0}^{1} r G(t r) d t\right|=\int_{0}^{1} G(t) d t=\int_{0}^{1} M(t) d t .
$$


Therefore the function $F$ will be unbounded if $\int_{0}^{1} u(r) d r=\infty$. Hence, by (2.11), we have the following result.

Theorem 3.1. Let $\Omega, \varphi, G$, and $F$ be as above. If

$$
\int_{1}^{\infty} \frac{d u}{\varphi(u) \exp \left\{\pi \int_{1}^{u} \frac{d x}{x \varphi(x)}\right\}}=\infty
$$

then $F(r) \rightarrow \infty$ as $r \rightarrow 1$.

Under the same assumptions on $\varphi$ and $\Omega$ as given above we can also formulate a converse to Theorem 2.4.

Theorem 3.2. Let $\Omega, \varphi, G$, and $F$ be as in Theorem 3.1, and define

$$
I_{p}=\int_{1}^{\infty} \frac{u^{p-1} d u}{\varphi(u) \exp \left\{\pi \int_{1}^{u} \frac{d x}{x \varphi(x)}\right\}} .
$$

If $I_{p}<\infty$ for $1 \leq p<q$ and $I_{q}=\infty$, then $F^{\prime} \in H^{p}$ for $p<q$ but $F^{\prime} \notin H^{q}$. Proof. That $F^{\prime} \in H^{p}$ for $p<q$ is the result of Theorem 2.4. In particular since $I_{1}<\infty, F$ is bounded from below by a constant $m$ (Theorem 2.2). If $I_{q}=\infty$, then $\int_{0}^{1} M^{q}(t) d t=\infty$ because of (2.16) and (3.2). By a theorem of Hardy and Littlewood [2, p. 87] this implies that $G \notin H^{q}$. Now $\left|F^{\prime}(z)\right|=$ $|F(z)| \cdot|G(z)| \geq m \cdot|G(z)|$, so we also get that $F^{\prime} \notin H^{q}$.

\section{EXAMPLES}

As we have commented on before, the conditions that we have given in terms of convergence of certain integrals are linked to how close $\Omega$ is to a halfplane. The examples that are given below will shed further light on this fact.

Example 1. Let $F(z)=1 /(1-z)^{\alpha}, \alpha>0$. Then $G(z)=F^{\prime}(z) / F(z)=$ $\alpha /(1-z)$. In this case $\Omega$ is the halfplane $\operatorname{Re} w>\alpha / 2$, and the function $F$ is certainly unbounded in $\Delta$.

The critical situation is of course when $\Omega$ opens up to be more and more like a halfplane, which means that $\varphi(r) \rightarrow \pi$ as $r \rightarrow \infty$. The condition (2.12) basically says that $\varphi$ cannot approach $\pi$ too fast. This is seen most clearly in the stronger condition $(2.13)$ where, to get integrability, the ratio $\pi / \varphi(u)$ must not go to 1 too fast.

Example 2. Let

$$
\varphi(t)=\frac{\pi t^{\alpha}}{A+t^{\alpha}}, \quad A>0, \quad \alpha>0 .
$$

Then $\varphi(t) \rightarrow \pi$ as $t \rightarrow \infty$. Computing we find that

$$
E(r)=\pi \int_{1}^{r} \frac{d t}{t \varphi(t)}=\log r+\frac{A}{\alpha}\left(1-\frac{1}{r^{\alpha}}\right)<\log r+\frac{A}{\alpha} .
$$

So

$$
\int_{1}^{\infty} \frac{d r}{\varphi(r) \exp \{E(r)\}}>\frac{K}{\pi} \int_{1}^{\infty} \frac{\left(A+r^{\alpha}\right) d r}{r^{\alpha+1}}>\frac{K}{\pi} \int_{1}^{\infty} \frac{d r}{r}
$$

where $K=\exp \{-A / \alpha\}$. Hence the integral diverges. The function $\varphi$ satisfies the conditions of Theorem 3.1 and therefore, if the corresponding domain $\Omega$ is symmetric about the real axis, the function $F$ will not be bounded. 
Example 3. Next we consider

$$
\varphi(t)=\frac{\pi \log t}{A+\log t}, \quad A>0,
$$

for $t \geq 2$. This function also satisfies the conditions of Theorem 3.1. Now

$$
E(r)=\int_{2}^{r} \frac{A+\log t}{t \log t} d t=\log r+A \log (\log r)-K .
$$

Further we get

$$
\int_{2}^{\infty} \frac{d r}{\varphi(r) \exp \{E(r)\}}=A C \int_{2}^{\infty} \frac{d r}{r(\log r)^{A+1}}+C \int_{2}^{\infty} \frac{d r}{r(\log r)^{A}}
$$

for some constant $C$. The first integral above converges for all $A>0$, whereas the second converges if and only if $A>1$. So in this case we get bounded functions $F$ depending on the value of $A$. With the same $\varphi$,

$$
\int_{2}^{\infty} \frac{d r}{r^{\pi / \varphi(r)}}=\int_{2}^{\infty} \frac{d r}{r^{1+A / \log r}}=e^{-A} \int_{2}^{\infty} \frac{d r}{r}
$$

This shows that the stronger condition (2.13) does not hold for this $\varphi$, no matter how large $A$ is.

Concerning the question of membership in $H^{p}$ spaces, we see that with this function $\varphi$ we get $F^{\prime} \in H^{1}$ for $A>1$ and $F^{\prime} \notin H^{p}$ for $p>1$. If we modify $\varphi$ to

$$
\varphi(t)=\frac{\alpha \pi \log t}{A+\log t}, \quad A>0, \quad 0<\alpha \leq 1,
$$

then $\varphi(t) \rightarrow \alpha \pi$ when $t \rightarrow \infty$, and a similar computation shows that then we get $F^{\prime} \in H^{p}$ for $p \leq 1 / \alpha$ if $A>\alpha$.

The methods developed in this paper can also be applied to classes of normalized starlike functions. In that case $g(z)=z f^{\prime}(z) / f(z)$ and $g(z)$ lies in some domain $\Omega$ in the right halfplane. The next example deals with such functions.

Example 4. In [6] the second author studied classes of normalized starlike functions with $z f^{\prime}(z) / f(z)$ lying inside a parabola. It was shown that these functions were bounded and that $f^{\prime} \in H^{2}$. With the notation we have used we now have

$$
\varphi(t)=2 \tan ^{-1} \frac{1}{\sqrt{A t}}, \quad A>0 .
$$

Then

$$
E(r)=\frac{\pi}{2} \int_{1}^{r} \frac{d t}{t \tan ^{-1} \frac{1}{\sqrt{A t}}}>\frac{\pi \sqrt{A}}{2} \int_{1}^{r} \frac{d t}{\sqrt{t}}=\pi \sqrt{A}(\sqrt{r}-1) .
$$

Putting this into (2.15) we get for some constant $K$ that

$$
\int_{1}^{\infty} \frac{r^{p-1} d r}{\varphi(r) \exp \{E(r)\}}<\frac{K}{A^{p}} \int_{\sqrt{A}}^{\infty} \frac{v^{2 p-1} d v}{\tan ^{-1}(1 / v) \exp (v / \sqrt{A})} .
$$

For large enough $v, \tan ^{-1} 1 / v>C / v$ for some constant $C$, so the integral on the right-hand side of the above inequality behaves essentially as

$$
\int_{\sqrt{A}}^{\infty} \frac{v^{2 p}}{e^{v / \sqrt{A}}}
$$


which converges for every $p$ and every $A$. This means that these starlike functions satisfy $f^{\prime} \in H^{p}$ for all $p>0$. In the case of $S S_{\alpha}^{*}(\alpha<1)$ we have $\varphi(t)=\alpha \pi$, and we immediately see from Theorem 2.3(b) and Theorem 2.5(b) that all these functions are bounded and that $f^{\prime} \in H^{p}$ for $p<1 / \alpha$, in accordance with results in [1].

Remark. Let $\Omega$ be a domain in the right halfplane such that (2.4) holds, and define $S^{*}(\Omega)$ to be the class of functions $f$ with $f(0)=f^{\prime}(0)-1=0$ and $z f^{\prime}(z) / f(z) \in \Omega$. Then all functions in $S^{*}(\Omega)$ will be bounded starlike functions with derivatives in $H^{1}$. Therefore they will all have an integral representation

$$
f(z)=\int_{|x|=1} \log \frac{1}{1-x z} d \mu(x)
$$

for some measure $\mu$. (See [5] and [6] for details.) Furthermore, since $f^{\prime} \in$ $H^{1}$, the boundary of $f(\Delta)$ will be a rectifiable Jordan curve. In [6] these results were obtained in the case that $\partial \Omega$ is a parabola, so this is a considerable generalization.

\section{REFERENCES}

1. D.A. Brannan and W.E. Kirwan, On some classes of bounded univalent functions, J. London Math. Soc. (2) 1 (1969), 431-443.

2. P.L. Duren, Theory of $H^{p}$ Spaces, Academic Press, New York and London, 1970.

3. $\frac{}{1983}$, Univalent functions, Springer-Verlag, New York, Berlin, Heidelberg, and Tokyo, 1983.

4. M.A. Evgrafov, Analytic functions, Dover, New York, 1978.

5. R.A. Hibschweiler and T.H. MacGregor, Univalent functions with restricted growth and Cauchy-Stieltjes integrals, Complex Variables 15 (1990), 53-63.

6. F. Rønning, Integral representations of bounded starlike functions, Ann. Polon. Math. (to appear).

Department of Mathematics and Statistics, State University of New York at Albany, Albany, New York 12222

Trondheim College of Education, Rotvoll allé, N-7005 Trondheim, Norway 